\title{
Analysis and Design of N-Ram Digital Games for problem-based solving for Early Childhood Geometry Learning
}

\author{
Taufik Ridwan ${ }^{1}$, Endang Hidayat ${ }^{2}$ \\ \{taufikridwan@upi.edu ${ }^{1}$ \} \\ Study Program of System and Information Technology Education, Universitas Pendidikan Indonesia, \\ Indonesia ${ }^{1}$ \\ Study Program of Elementary School Teacher Training, Universitas Pendidikan Indonesia, \\ Indonesia $^{2}$
}

\begin{abstract}
Development and growth in children must be stimulated properly in order to occur optimally. In the phase of growth and development, children spend most of their time playing. Generally, children have high learning abilities and curiosity. Today, children have started to become familiar with gadgets and digital games. Unfortunately most of the games that are played more suitable for adults. Not infrequently due to lack of parental supervision and lack of choice to play make children addicted to games that are not suitable for them. Actually, in this phase children need to get interesting positive stimulus to hone their intelligence. For that condition, one of many solution is the availability of games for learning media. This paper tries to analyze and design prototypes of N-Ram Digital Games for children. N-Ram digital games are games for learning geometry in children. The method used in this research is Research and Development (R\&D) with a waterfall approach in Software Development. The result of the analysis and design of this prototype is N-Ram Digital Games to learn geometry through pairing and adjusting images accordingly, and building a geometric shape from several available geometric shapes.
\end{abstract}

Keywords: N-Ram, Learning media, Geometry, Children

\section{Background}

Development and growth in early childhood must be stimulated properly in order to occur optimally. This phase is often called the golden age of growth and development. One of the developments that must be stimulated is cognitive development by introducing objects around the child. Basically in growth and development, children can not be separated from objects that are around in play. Since childhood, they have been familiar with objects in various shapes and forms, including geometric shapes such as coins, cabinets, tables, books, balls, or other objects that are used to meet the needs of everyday life for playing purposes

Geometry is part of the material that studies the concepts of form and space. Some reasons why geometry needs firstly to be taught are firstly, geometry is the only field of mathematics that can link mathematics with the physical form of the real world. Secondly, geometry is the only field of mathematics that can enable mathematical ideas to be visualized, and thirdly, geometry can provide non-singular examples of mathematical systems[1]. 
Learning to recognize geometric shapes will help children to understand, describe, and describe objects that are around them. Introducing geometric shapes in early childhood can be started from building the concept of geometry by identifying the characteristics of geometric shapes. The ability to recognize geometric shapes in children is always associated with learning mathematics. Mathematics in playgroup is an activity of learning about mathematical concepts through play activities in daily life and is scientific. Learning as well as playing is one way that can be used for learning geometry mathematics in children.

Game research for learning mathematics has actually been done. Lin et al try to develop a collaborative and manipulative virtual Tangram puzzle to facilitate children to learn geometry in the computer-supported collaborative learning environment with Tablet PCs. The results suggest that children's competency in rotation and space of shapes had been improved and the scores gap between lower and higher achievers had been narrowed[2]. Liu examines the use of games where there is an increase in children's ability in learning mathematics with accompanying play activities compared to conventional learning in the classroom[3].

The rapid development of technology has influenced children by playing. In this phase of growth and development, children spend most of their time playing. Children in general have the ability to learn and curiosity is very high. They tend to like games that attract attention to be played. The presence of the gadget has attracted the attention of children to try and even become active users. The gadget presents a container filled with various multimedia in one device. This complete feature is not obtained by children in other media, so the tendency to like gadgets is very high. The impact, not infrequently children prefer to play gadgets rather than other activities including learning. The worst thing is sometimes making children become addicted to games.

An approach needs to be found that can help children to be more wise in using gadgets but does not prevent children from playing it. One of them is a digital game that can stimulate children's intelligence. This has finally led to the idea of how to make children go through the process of learning in play activities, so that children will still get entertainment through an interesting and fun media.

From two previous studies[2],[3], found children are interested in learning while playing. Geometry games on Android Playstrore actually already exist. One of them is the tangram game in various versions. But from some of the tangram games available in playstore, most of the tangram that are general in nature are even more suitable to be played for adults rather than specifically for young children. This is based on the difficulty that is quite complicated in playing and completing the available games. One that can be used as a solution is the availability of children geometry games that are useful and according to the level of difficulty with the age of the player.

This research tries to provide alternative solutions in the form of digital games to help children to learn. Children can feel fun while learning simultaneously. This research will try to provide a medium for learning starting from the phase of knowing until to apply geometric shapes.

$\mathrm{N}$-ram game that is designed is a game that utilizes collaborative science learning with technology combined with mathematics or better known as STEM. N-Ram combined with STEM must produce a fun children's learning media. 


\section{Literature Review}

\subsection{Geometry learning mathematics for children}

Mathematics is a discipline of logic for mastering the form, composition, quantity, and concepts related to one another with the number of which is divided into three main areas, namely algebra, analysis, and geometry. Mathematics is one of the sciences that is widely used in everyday life. Both in general and specifically. Almost in every aspect of life, mathematics can be applied.

Geometry is a part of mathematics that deals with points, lines, fields and space. Space is a set of points that can form geometric shapes, lines are a set of parts of space that are sets of points that have special properties. Fields are sets of points that lie on a flat surface

Learning geometry at an early age is possible to teach even though it has to be more creative and realistic. Geometry is considered to have many applications in mathematics and real life, which also contain many elements of problem solving. Mathematics learning especially geometry for early childhood aims to recognize, point, mention and collect objects around based on geometric shapes. The first stage a child learns geometry is topological. There are five stages of children's learning geometry, which are as follows:

1) Introduction Stage. At this stage students are already familiar with geometric forms, such as triangles, cubes, balls, circles, and other things, but they have not yet understood their properties.

2) Analysis Phase. At this stage, students can already understand the properties of concepts or geometric shapes. For example, students know and recognize that the opposite sides are the same length, that the lengths of the two diagonals are the same length and intersect each other the same length and so on.

3) Ordering Phase. At this stage, students are able to recognize geometric shapes and understand the properties and he can already sort the geometric shapes related to each other

4) Education Stage. At this stage, his deductive thinking has begun to grow, but has not yet developed properly. Mathematics is a deductive science, because conclusions are drawn up, the proof that has to be done deductively. At this stage, students can already understand the importance of deductive conclusions, because for example he can see that the conclusions drawn inductively may be wrong.

5) Accuracy Stage (Ringor). At this stage, students can understand that the accuracy of the fundamentals is important.

Although virtually all young children have the capability to learn and become competent in mathematics, for most the potential to learn mathematics in the early years of school is not currently realized. This stems from a lack of opportunities to learn mathematics either in early childhood settings or through everyday experiences in homes and in communities[4].

The solution about that problem one of them is educator's willingness to make math games for children. In the math game, there is a game called tangram. This game is a square puzzle game that is cut into 7 parts ( 2 in the form of a large triangle, 1 in the form of a square, 1 in the shape of a ladder, 1 in the form of a medium triangle, and 2 in the form of a small triangle), follow Figure 1. 


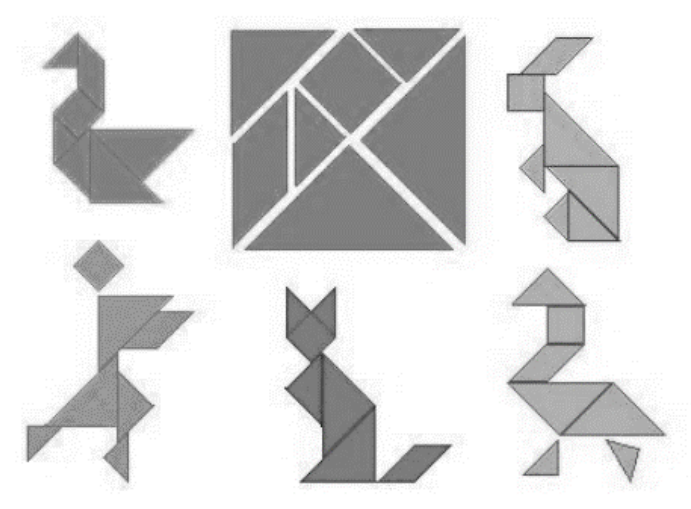

Fig. 1. Example of Tangram [3].

\subsection{Digital Games As Education Methods On Early Childhood}

Education for early childhood is the most important aspect that must be given, because competency growth and development requires maximum guidance. The world of children will not be separated from play and imaginative. So that the method of education provided is more for play and imagination. These two elements are packaged and conceptualized into the education curriculum, and are part of the theory of education for early childhood.

The National Education Association for Young Children (NAEYC) identifies early childhood as a group of individuals who are in the age range between $0-8$ years. According to Law Number 20 Year 2003 concerning the National Education System Article 1 number 14 states that Early Childhood Education (PAUD) is a coaching effort aimed at children from birth to the age of six years which is carried out through the provision of educational stimuli to help physical growth and development and spiritually so that children have readiness to enter further education. So early childhood education focuses on laying the basis for the growth of physical development, attitudes, intelligence on intellectuals, communication through language vocabulary, and so forth.

Education for children is very different in treatment with adults and requires several stages, including: oriented to the needs of children, learning through play, having a conducive environment, using educational media, continuous learning, and using representative learning. Children's education is based on having fun, while education for adults puts forward the seriousness. These different learning patterns must be considered by educators.

Literally an educational method for children to orientation on play. Especially with the development of technology, the more complete the need to play in children. Children will feel comfortable with the presence of technology in the form of gadgets, so they don't know the time and sometimes get angry when they take the gadget. A suitable method for children's education is to utilize the advantages of technology to improve children's abilities. Collaboration between educators and gadgets is the right way, considering that children prefer to play using the technology. The use of gadgets as an interactive learning media is an alternative and the right choice to improve children's competence. Besides the child's visual abilities will increase by itself depends on continuity in play.

Mobile game is a game that can be run on a cell phone or cellular, so users can play it portable [5]. The term educational game basically comes from English, namely Game and 
Education Collaboration on the two words is expected to be able to provide insight to users to learn effectively and persuasively. Salem Alanazi in his research shows that electronic-based games can provide motivation to learners [6].

Allsop \& Jessel conducted research to teachers to determine perceptions of learning based on digital games and their effects on students for learning achievement. In addition, Allshop \& Jessel also examined the role of digital game-based learning in supporting curriculum and learning culture in the UK and Italy. The results of this study indicate that teachers are very concerned about the application of technology and its rules when applied to children. Pedagogic curricula and practices also require flexible models for the application of digital games in elementary school classes [7].

The results of the two studies above, show that the role of digital games can help learning for children, especially early childhood. The use of Games for children should require supervision from parents and teachers so that they are not excessive and can be used as part of positive learning media.

\section{Research Methods}

The method used in this research is Research and Deptovment (R\&D). This research intends to combine fun learning with technological development. The output of the research will produce a prototype game product for the process of helping learning geometry for children.

In the stages carried out in making games using this R\&D method, a software design method called waterfall is used follow at Figure 2. The stages that are traversed by the waterfall will be useful to be able to produce reliable and effective software. This method is a method in software design that starts from software requirements to analysis requirement of user until the software can be maintain[8].

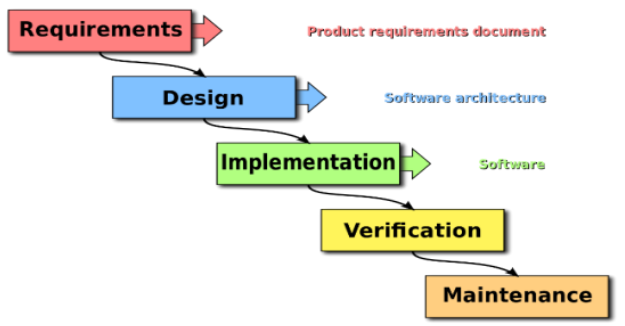

Fig. 2. Methode Of Waterfall On Software Engineering.

The process of making software that is passed is the phase of needs requirement, Design, implementation (making a program with coding process) verification/ testing, and maintenence operating the software.

In the realm of mathematics, there are several traditional games that are well known to the wider community and can be used to stimulate intelligence in child development. One such game is Tangram. Tangram comes from the words Tang and Gram, which means seven magic pieces. In the modern world, this game is known as the Chinese puzzle. Tangram game consists of various geometric shapes, for example squares, rectangles, triangles, squares, and various other geometric shapes seen at Figure 3. Usually the shapes of objects in this game 
consist of various colors. The pieces are in the form of a flat shape called a tan and when combined they will form a square. This puzzle aims to create a specific shape using all available shapes and should not overlap.
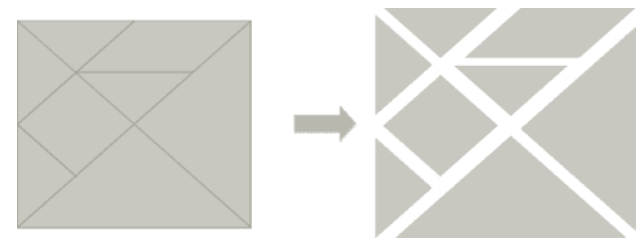

Fig. 3. How to Play Tangram.

Games that will be built in this study are N-Ram. N-Ram is a digital game modified by researchers from the tangram game. In the tangram game, the number of geometric structures constructed is only seven. But in this N-Ram the number of geometric structures can be less or more than seven. This game is intended for early childhood, so that at the lowest level of the game, the difficulty level is not difficult. The aim of N-Ram digital games is to solve geomterial problems. The problem raised is in the form of matching shapes, arranging pieces of shapes to the process of forming a space structure.

The main target of designing board games is children aged 3-10 years who are still sitting in kindergarten, or elementary school. However, it is possible for this game to be played by all groups. The main requirements are curiosity, the desire to learn, and try new things. From this analysis then some analysis is carried out in the making of N-Ram games as follows:

The big idea to be achieved is to make educational games. The results of initial observations found that children are not maximized in recognizing and understanding geometric shapes, but actually there are games that can help learn them. Conventional and digital games already exist but cannot be used optimally by early childhood because of difficulties which are quite difficult to play by early childhood. the contribution of this research is the development of instructional media through digital games. The end result of games that are built aside from being able to be used as learning media can also be used for entertainment or play media for children and adults.

In this game, players must be able to match shapes, colors, or arrange puzzle pieces to become whole depending on the level. Children will be tested for understanding geometry in a fun way. The reward of this game is in the form of player points in completing challenges within a certain time. The faster the player completes each round, the more points are won. The penalty that is received when a player is wrong is the reduction in the stars used for the game. When the star is zero, the game is considered over.

Analysis of system modeling is how to describe the model of data flow and functional systems when running. The modeling used in this research is Unified Modeling Language.

1) Use Case Diagram. In general, use case diagrams for N-Ram games that are built can be seen in Figure 4. 


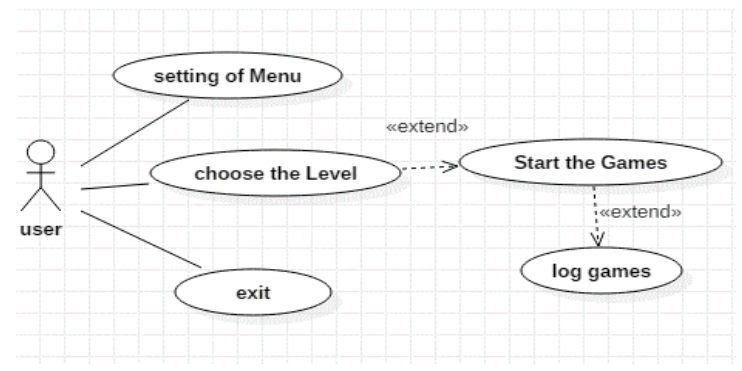

Fig. 4. Analysis of Use Case Diagram.

From Figure 4 the system can be explained that there are 3 activities or something that can be done, namely the settings menu, select a level, and exit. When a user selects a level, it will automatically be taken to another activity, which is to start playing.

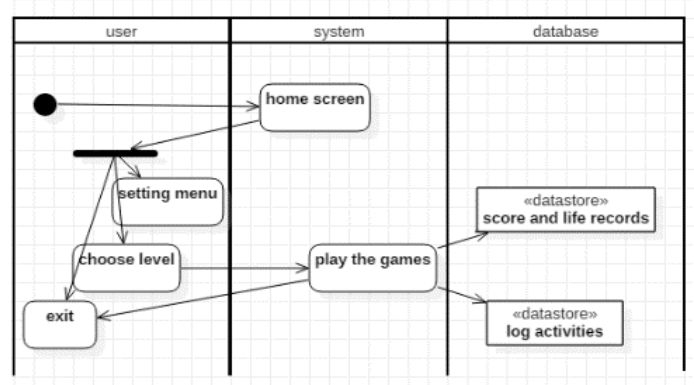

Fig. 5. Analysis of Activity Diagram.

From Figure 5 you can see everything that is an activity in this game. This game is generally in the home menu, the user can choose 3 options namely set the game option that contains information and settings for this game, then select the level to play at which level, and exit the game.

Games that will be made in this research are mobile based. Games run on an android operating system. When a player plays, the player must carry out the commands given per level. Players will click on the image or move the puzzle piece. The higher the level of the game, the level of the game is also more difficult and less time is provided by the system.

\section{Result and Discussion}

From the results of the analysis of the system that has been built then made a design of the N-Ram user interface games per stage as follows Figure 6. 

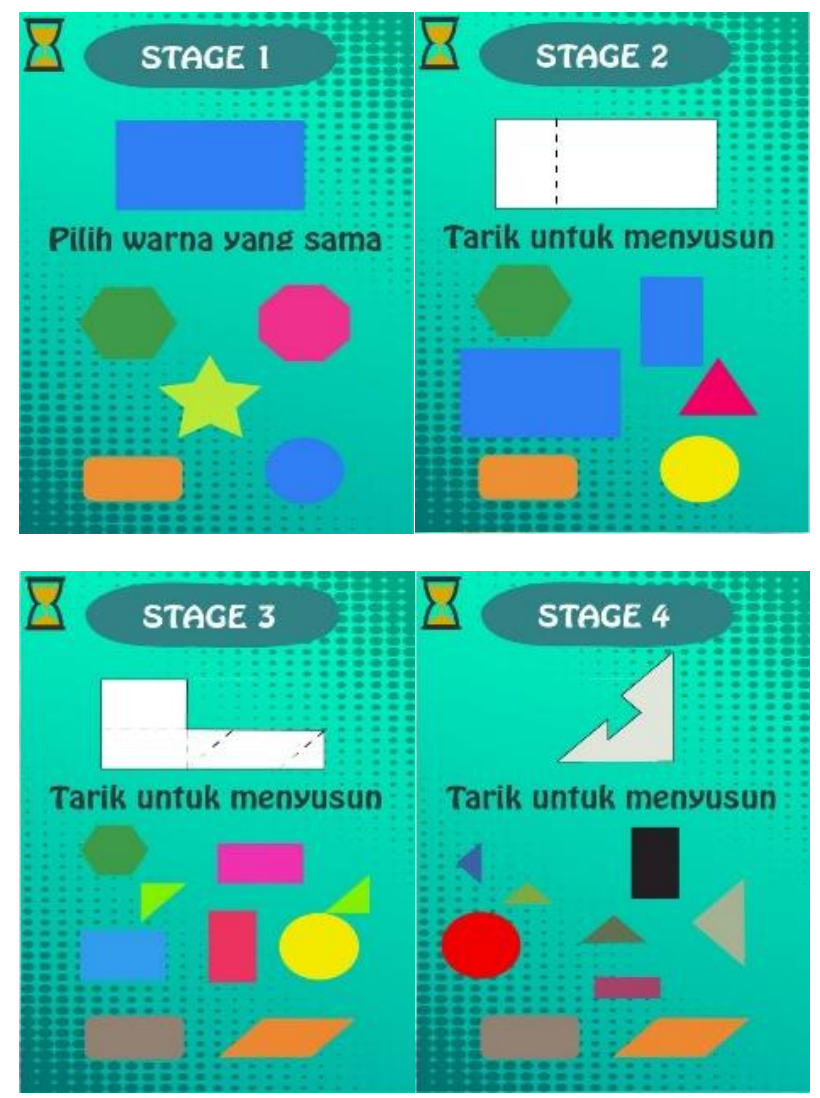

Fig. 6. Design user interface N-Ram

From Figure 6, it can be seen that each game has increasingly difficult levels. It aims to stimulate children to play from the easiest to the rise per round becomes increasingly difficult but is still adapted to the ability of children.

\section{Conclusion}

Child's growth and development period is a time when children like to play and imitate things that are around. The rise of games aimed not at children but played by children is a sad thing. Education and technology activists should be able to answer these opportunities and challenges. Games for children should be able to stimulate children to help improve their ability to learn.

$\mathrm{N}$-ram games that are designed are a modification of the tangram game but adjust the challenges per round according to the ability of early childhood. N-Ram Digital Games try to package learning geometry into fun and educational games. 
The result of the analysis and design of this prototype is N-Ram Digital Games to learn geometry through pairing and adjusting images accordingly, and building a geometric shape from several available geometric shapes.

\section{References}

[1] Usiskin, Z.: Van Hiele Levels and Achievement in Secondary School Geometry. (Final report of the Cognitive Development and Achievement in Secondary School Geometry Project.) Chicago: University of Chicago. (ERIC Document Reproduction Service No. ED220288). (1982)

[2] Lin, Chiu-Pin; Shao, Yin-juan; Wong, Lung-Hsiang; Li, Yin-Jen; Niramitranon, Jitti.: Turkish Online Journal of Educational Technology - TOJET, v10 n2 p250-258 Apr 2011: The Impact of Using Synchronous Collaborative Virtual Tangram in Children's Geometric. (2011)

[3] Liu, Yuting.: Tangram Race Mathematical Game: Combining Wearable Technology and Traditional Games for Enhancing Mathematics Learning. Worcester Polytechnic Institute. (2014)

[4] Cross, C. T., Woods, T. A., \& Schweingruber, H.: Mathematics learning in early childhood: Paths toward excellence and equity. Washington, DC, US: National Academies Press. (2009)

[5] Shiratuddin, N., \& Zaibon, B.S.: International Journal of Mobile Learning and Organisation, 4(1): Mobile game-based learning with local content and appealing characters. (2010)

[6] Alanazi, T.S.: International Journal of Humanities and Social Science, 7(1): The Efficiency of Using Educational Electronic Games in Improving English Language Skills for Fourth Elementary Grade Students. (2017)

[7] Allsop, Y., \& Jessel, J.: International Journal of Game-Based Learning, 5(1), 1-17: Teachers' Experience and Reflections on Game-Based Learning in the Primary Classroom: Views from England and Italy. (2015)

[8] Pressman, R.S.: Software Engineering: a practitioner's approach. New York: McGraw-Hill. (2010) 